\title{
Using Open Source, Open Data, and Civic Technology to Address the COVID-19 Pandemic and Infodemic
}

\author{
Shinji Kobayashi', Luis Falcón ${ }^{2}$, Hamish Fraser ${ }^{3}$, Jørn Braa ${ }^{4}$, Pamod Amarakoon ${ }^{5}$, \\ Alvin Marcelo ${ }^{6}$, Chris Paton \\ 1 National Institute of Public Health, Saitama, Japan \\ 2 GNU Solidario, GNU Health, Las Palmas de Gran Canaria, Spain \\ 3 Brown Center for Biomedical Informatics, Brown University, Providence, USA \\ ${ }^{4}$ University of Oslo, Oslo, Norway \\ 5 University of Colombo, Colombo, Sri Lanka \\ ${ }^{6}$ Asia eHealth Information Network, Manilla, Philippines \\ 7 Nuffield Department of Medicine, University of Oxford, Oxford, UK
}

\begin{abstract}
Summary
Objectives: The emerging COVID-19 pandemic has caused one of the world's worst health disasters compounded by social confusion with misinformation, the so-called "Infodemic". In this paper, we discuss how open technology approaches - including data sharing, visualization, and tooling - can address the COVID-19 pandemic and infodemic.

Methods: In response to the call for participation in the 2020 International Medical Informatics Association (IMIA) Yearbook theme issue on Medical Informatics and the Pandemic, the IMIA Open Source Working Group surveyed recent works related to the use of Free/Libre/Open Source Software (FLOSS) for this pandemic.

Results: FLOSS health care projects including GNU Health, OpenMRS, DHIS2, and others, have responded from the early phase of this pandemic. Data related to COVID-19 have been published from health organizations all over the world. Civic Technology, and the collaborative work of FLOSS and open data groups were considered to support collective intelligence on approaches to managing the pandemic.

Conclusion: FLOSS and open data have been effectively used to contribute to managing the COVID-19 pandemic, and open approaches to collaboration can improve trust in data.
\end{abstract}

\section{Keywords}

COVID-19, Public Health, Open data, Free/Libre/Open source software

Yearb Med Inform 2021:38-43 http://dx.doi.org/10.1055/s-0041-1726488

\section{Introduction}

The new coronavirus infection was first reported in Wuhan, China in December 2019 and outbreaks spread rapidly all over the world. The SARS-CoV-2 virus, and COVID-19, the resulting disease, has caused one of the most rapidly developing health disasters in generations. The World Health Organization (WHO) declared it a "pandemic" on March 11 [1]. As of January 23, 2021, the WHO dashboard reported there had been 92,098,879 confirmed cases of COVID-19, including 2,098,879 deaths in the globe [2].

As the disease became more prevalent, populations became increasingly concerned and social unrest has been reported including protests against non-pharmaceutical interventions such as "lockdowns". Misinformation related to COVID-19 propagated through mass media reports and social networks and risk causing panics or undue complacency exacerbating the health consequences of the disease [3]. In the United States, a comment from President Trump about injecting disinfectant misled people into drinking the disinfectant [4]. In Japan, face masks became scarce from February 2020, and toilet paper disappeared due to panic buying because of the mistaken belief that they were made from the same paper as masks. The International Telecommunication Union has officially denied the rumors that the $5 \mathrm{G}$ network was associated with the spread of COVID-19 [5]. "Infodemics" is a term, which is defined as the rapid spread of information of all kinds, including rumours, gossip and unreliable information related to epidemics [6]. From the early stage of COVID-19 pandemic, WHO's director general, Tedros Adhanom Ghebreyesus said "We're not just fighting a pandemic; we're fighting an infodemic" (2020 Munich security conference [7]). WHO and public health organizations of each country are struggling to ensure that publishers provide accurate information on their websites and media to avoid repeating and spreading such misinformation $[8,9]$. In addition, the infodemic has caused discrimination and prejudice against people of Asian origin and stigmatization of those infected with COVID-19 and health workers $[10,11]$. Open source software and open data initiatives can provide more accountability and transparency of data sources, helping to combat the secrecy of algorithms and data sharing typical of many social media platforms.

Our working group has been studying free/libre/open-source software (FLOSS) in the medical field and extended the scope to open data and open science $[12,13]$. From the COVID-19 pandemic and infodemic, we have found that open-source and open data collaborations, termed "Civic Technology", worked efficiently against COVID-19, and report this concept as a working group paper here. 


\section{FLOSS Projects Responses to COVID-19}

As we have previously reported, FLOSS projects are increasingly utilized in the medical domain [14-16]. During the COVID-19 pandemic, FLOSS groups have responded from the early stages, building on large installed bases in many Low and Middle-Income Countries (LMICs) with experience from dealing with previous epidemics including Ebola. Well established examples are described below (Table 1).

\subsection{GNU Health}

GNU Health is a Libre digital health ecosystem from the NGO GNU Solidario [17]. GNU Health is an international, community-driven project that provides the tools for individuals, health professionals, institutions and governments to proactively assess and improve the underlying determinants of health, from the socioeconomic agents to the molecular basis of disease [17]. It manages the internal processes of a health institution, such as financial management, stock \& pharmacies and patient management. The ecosystem consists of different components, such as Electronic Medical Record (EMR); Hospital Management System (HMIS); Laboratory (LIMS); clinical genetics and analytics/epidemiology engine, among others. The Personal Health Record (PHR) component, MyGNUHealth, will be released in Q1 2021. GNU Health has been implemented in countries around the globe from primary care centers, research institutions, to large, national-level implementations.

GNU Health has developed the "Contact Tracing" package that is being used in the context of COVID-19. It's also useful in the control of other infectious diseases, such as Ebola or tuberculosis. The government of Entre Ríos, Argentina is currently using the contact tracing package to track COVID-19 cases in the public health institutions of Diamante [21].

The GNU Health Federation has been chosen as the COVID-19 real-time observatory by the government of Argentina. Argentina National Research and Development Agency (Agencia de Promoción de la Investigación, el Desarrollo Tecnológico y la Innovación (Agencia I+D+i)) chose Dr. Sassetti's project on GNU Health Federation as an Epidemiological Observatory [22].

Table 1 FLOSS response to COVID-19.

\begin{tabular}{|l|l|l|}
\hline & Description & COVID-19 response \\
\hline GNU Health & $\begin{array}{l}\text { Hospital Management / Health } \\
\text { information system [17,18] }\end{array}$ & $\begin{array}{l}\text { Contact tracing package } \\
\text { Epidemiological reporting } \\
\text { Laboratory, test management } \\
\text { Vaccination management }\end{array}$ \\
& & A new version for COVID-19 clinical \\
& management with HL7 \\
& Electronic health record [19] & Fast Healthcare Interoperability Resource \\
& & (FHIR) resources \\
& & Symptom screening form \\
& & Vaccine management \\
\hline OHIS2 & & Contact tracing \\
(District Health & Daily, weekly reporting of COVID-19 \\
Information Software) & system [18] & patients \\
& & Vaccine Delivery Toolkit \\
\hline LIFE & & New open access training scenarios on the \\
(Life-saving Instruction & Open access emergency training & management of COVID-19 patients \\
for Emergencies) & smartphone app [20] & \\
\hline
\end{tabular}

GNU Health provides an epidemiological surveillance report. Real-time epidemic curves and demographics to assist in studying the incidence and prevalence in many infectious as well as non-communicable diseases. The government can study the causes and the impact of these conditions on different groups of society, ranging from the molecular to the social determinants of health [23].

In April 2020, GNU Solidario and the GNU Health project partnered with the European Commission "EUvsVirus hackaton" as civil society and public authority partners, promoting, teaching and showing the benefits of Libre Software in the Public Health domain, particularly in the context of the COVID-19 pandemic [24].

\subsection{OpenMRS}

OpenMRS is an open source, standards-based and modular electronic health record (EHR) used clinically in at least 44 LMICs [19, 25]. The main focus has been on the care of infectious diseases including human immunodeficiency virus (HIV) and tuberculosis (TB), with versions also supporting care delivery for primary care, maternal and child health, heart disease, cancer, and surgery. It has been used for management of acute epidemic diseases in the past including cholera in Haiti, and a custom version was created for use in an Ebola treatment center in Sierra Leone in 2015. This had a tablet interface designed for use by staff with full personal protective equipment (PPE) [26].

Several mHealth platforms have been adapted to support epidemic management including Ebola and COVID-19 such as CommCare [27], Medical Mobile [28] and Open Data Kit (ODK) [29], providing effective support for screening and contact tracing in the community. To ensure a complete picture of the disease impact and management these systems need to work synergistically with EHRs in health facilities. The EHR can capture clinical presentations of COVID-19 patients in primary or urgent care, their clinical treatment, progress and longer term follow up. OpenMRS has a strong focus on open standards and interoperability using a concept dictionary mapped to ICD10, SNOMED CT and LOINC [30]. A new 
module supports the latest versions of the HL7-FHIR (Fast Healthcare Interoperability Resources) interoperability standard [31] and has been adapted to link OpenMRS instances to the OpenELIS laboratory information system in Haiti.

Early work has been carried out to adapt OpenMRS to support screening, testing and management of COVID-19, with initial deployments in Nepal, Haiti and Kenya. In Nepal, Possible Health uses the Bahmni version of OpenMRS. They have added a screening note to help clinicians assess patients presenting with COVID symptoms or assess exposure risk. It was designed in consultation with official guidelines and physicians who already use NepalEHR [32]. In Haiti, at the University Hospital of Mirebalais, Partners in Health and its sister organization in Haiti, Zanmi Lasante, have added COVID-19 testing protocols to the OpenMRS order entry system along with basic care forms and are deploying clinical forms for managing patients admitted to the hospital. In Kenya, the ministry of health along with Kenyan and international collaborating organizations have developed and deployed a version of the "KenyaEMR" OpenMRS system at national level to collect, manage and report COVID-19 case data. This includes data from mobile health systems and links to District Health Information Software 2 (DHIS2) for reporting.

The OpenMRS community have worked together to develop core tools to support management of COVID-19. These include:

- Release of new versions of the OpenMRS concept dictionary with concepts for screening, testing and clinical management. These have been mapped to HL7 FHIR profiles led by Dr Andy Kanter;

- Creation of WHO COVID-19 screening forms in OpenMRS, and development of the new FHIR module led by a team at Brown University (Providence, USA);

- Collaborative work with VecnaCares inc on improving interoperability of OpenMRS with the District Health Information System - DHIS2 (see below) to allow rapid reporting to district and national levels from OpenMRS.

Goals for the next year are to support interoperability with mHealth applications using FHIR, to improve decision support for
COVID-19 management, and to improve the quality of data collection, coding and export from OpenMRS to facilitate rapid surveillance, prediction modelling and clinical research. OpenMRS has several implementations that support vaccine management, primarily for children, these offer platforms for rapid scale up of Sars-Cov-2 vaccines administration including tracking patients for second doses and any adverse events.

\subsection{Sri Lanka and DHIS2 Digital COVID-19 Response}

The COVID-19 digital response package using the DHIS2 open source software platform [18] was rapidly developed following the pandemic outbreak, with components for registration of cases, contact tracing, reporting daily and weekly summaries and more. A configuration package for DHIS2, which can easily be downloaded and adapted to meet individual country needs, was released early March (dhis2.org/covid-19). More than 60 countries are using DHIS2 and the intention was to leverage this existing infrastructure and country capacity for rapid deployment.

Sri Lanka was the first country to deploy DHIS2 for COVID-19 response. The Ministry of Health in Sri Lanka foresaw the threat of the pandemic from incoming tourists and decided to develop an integrated digital surveillance system, which was designed within five days based on the DHIS2 platform utilizing the country's existing capacity. There was collaboration with the HISP group supporting DHIS2 development at University of Colombo as well as the government's information, communication and technology (ICT) agency in this process. The system, which was approved by the higher administration from the ministry of health, was rapidly implemented following brief training. With the change of the disease landscape and spread, new requirements emerged necessitating development of new modules to the existing system which were not achievable by customizing DHIS2. The ministry decided to develop additional components on the DHIS2 platform and launched a two-day hackathon to obtain contributions from voluntary software developers to design web apps which could be installed on exist- ing DHIS2 platform. The ICT Agency of the Government announced the requirements on their social media networks and volunteer developers, mainly from the private sector, contributed their time and efforts for the worthy cause of producing a product to monitor COVID status of the country. For the initial two days, over 20 of them volunteered and the effort continued at full pace at least for a period of two months during which several apps on DHIS2 platform were developed in addition to a mobile application for citizens. The web apps produced during the hackathon included contact mapping, visualization, and intensive care unit bed management modules. Integration components to address bottlenecks in implementation were also designed, such as integration with the immigration information system. With expanding requirements, several other modules were customized such as quarantine, case management and laboratory modules. The collaboration with the global community facilitated obtaining rapid support from DHIS2 core development team for the efforts in the hackathon. Metadata and modules developed in Sri Lanka were shared with the global DHIS2 community and were quickly adopted by many countries across the world.

Thus, the agility of response to changing requirements supported by the open source platform with a global community, existing local capacity and multisector collaboration resulted in a successful digital surveillance system in a LMIC amidst an ongoing pandemic.

\subsection{Life-saving Instruction for Emergencies (LIFE)}

The LIFE project is a collaboration between the University of Oxford and the KEMRI-Wellcome Trust Research Programme in Nairobi, Kenya [20]. The project has developed smartphone apps and immersive VR training experiences to teach healthcare workers in Africa how to manage medical emergencies. The smartphone apps are released as open access with free downloads through app stores to the user's own smartphone. LIFE VR is accessible for free through the ENGAGE VR platform [33]. After downloading the app, the user is presented with a series of medical emergencies that they manage by navigating 
through a realistic 3D environment, gathering together the correct pieces of medical equipment they will need, and then successfully performing the key steps needed to save lives in emergencies. LIFE was recently evaluated in a study in Kenya that showed significant learning gains between rounds of practice using the app [34].

In response to the COVID-19 pandemic, the LIFE team rapidly developed a series of COVID-19 open access training scenarios that use an interactive 3D hospital with virtual patients to teach clinicians how to manage patients suspected of being infected with the novel coronavirus. Users are taught to don and doff PPE and ensure that patients are isolated and treated in accordance with WHO guidelines. After completing the training, users are given feedback on any errors they have made and are able to repeat the training until they are proficient.

\subsection{Contact Tracing}

Digital contact tracing for COVID-19 patients and citizens was suggested to be effective to control the outbreak by the experience in Singapore and through mathematical modelling [35]. Both Apple and Google provide contact tracing API to their products, iOS, and Android. They limit access to the representative agency of each country to protect the privacy of users. The ethics and privacy issues around contact tracing are still controversial due to stigmatism of COVID-19 related patients and health workers [36, 37] Japan and Germany have also developed contact tracing software using the Google/Apple API as open-source software and made it available to the public to show their transparency [38, 39]. Limitation for the usage of data is also a problem that will be discussed in a later section of this article.

\section{6 openEHR Clinical Models and Digital Guidelines}

Zhejiang University has an openEHR based clinical repository of 1.5 million patients. They overlayed a COVID-19 screening system on the repository by developing openEHR templates [40]. The templates are shared in the domestic and international openEHR clinical knowledge repositories and have been utilized for monitoring patients and developing digital guidelines for COVID-19 patients [40].

\section{Open Data for COVID-19}

"Open data " is a movement that promotes publication of data with open licenses so that they are available "for anyone to use, for any purpose, at no cost" [41]. As we described in a previous working group paper, open data is also beneficial for healthcare [12, 42], particularly in the area of public health $[43,44]$. Using a policy of open data has been shown to help curb the Ebola [45] and Zika viruses [46]. From the early phase of COVID-19, data was made open by the Chinese government based on the lessons of SARS [47]. The complete sequence of SARS-CoV-2 was shared in GenBank and available for research, clinical test, drug/vaccine development [48]. Comprehensive lists of COVID-19 related data sets are accessible online [49, 50].

Johns Hopkins University developed an interactive website to visualize COVID-19 epidemics in China using ArcGIS and open data from the Chinese CDC [51]. They also provided open data on the number of confirmed COVID-19 cases and deaths. Using this data, a number of websites have been constructed for data visualization, analysis and decision-making. The data was shared in Google spreadsheets at first and is now published on GitHub. WHO also shares COVID-19 open data on their website [2] (that was itself developed as open-source software).

The merits of open data is not only availability, but the transparency of data and decision making could contribute to mitigate "infodemic" [52, 53].

The Department of Health of the Government of the Philippines releases COVID-19 data $^{1}$ on a regular basis. The data are culled from three data sources once a day, uploaded, and transformed in the cloud where field restriction and anonymization algorithms are applied. After the transformation in a cloud

https://drive.google.com/drive/folders/1ZPPcVU4M7T-dtRyUceb0pMAd8ickYf8o data warehouse, the data are loaded into a dashboard and into a data drop. The sources range from a case line list, testing aggregates (from laboratories), and a bed tracker (for hospitals and clinics). By making the data open, the government encourages more stakeholders to participate in the analysis and thus promotes transparency and innovation.

However, there are limitations for making health data open access [12]. Even though contact tracing is important in epidemiological investigations, personal identifiable data should not be opened and should only be shared within public health professionals on a restrictive basis. It is controversial that Singapore changed the data policy of contact tracing for criminal investigations $[54,55]$. WHO published an ethical guide for contact tracing apps that suggested prohibition of the use of personal data generated by the public health department [37].

\section{Civic Technology Movement}

"Civic technology (tech)" describes collaborations between governments and citizens using open data and FLOSS [56].

In Japan, the Tokyo Metropolitan Government commissioned a non-government organization, Code for Japan (CfJ), to build a website to display their open-data (https:// catalog.data.metro.tokyo.lg.jp/en/dataset/ t000010d0000000068) on COVID-19 on February 26. CfJ developed and launched the website (https://stopcovid19.metro.tokyo. lg.jp/en) on March 3 after only five days. The source code was opened in a GitHub repository that had gathered 12,798 commitments from 97 contributors as of October 62020 (https://github.com/tokyo-metropolitan-gov/ covid19). The site was translated into eight languages and the derived codes covered 30 of 47 prefectures in June, and 43 by October.

We surveyed the relationship between open data and open-source software for COVID-19 based on the Tokyo COVID-19 site at the end of June.

Table 2 shows the ratio of existing open data and deployment of visualization site (COVID-19 Dashboard) for COVID-19. Visualization sites for COVID-19 were deployed more in the prefectures that publish 
open data for COVID-19 (odds ratio 2.27, Fisher test: $p=0.00058$ ). This might suggest that open data by prefecture governments had promoted civic technology movements and better public access to critical health data in the pandemic.

In Taiwan, the government restricted the purchase of face masks by citizens due to the shortage of PPE. To avoid panic buying, the Taiwan government provided open data on real-time mask availability with the location of pharmacies from February 2020. This helped to support the creation of 110 software projects to effectively visualize face mask distribution on the map until the mid-March, 2020 [57].

\section{Conclusions}

In this pandemic and infodemic, the communities supporting FLOSS for medicine, GNU Health, OpenMRS, DHIS2, and LIFE, have all responded from the earliest phase. The WHO also started developing its COVID-19 dashboard as FLOSS [58]

There are many reasons why open data is thought to be effective to control epidemics: it allows effective communication of risk including identifying case increases, and local hot spots; it allows the calculation of the number of new infections per case ( $\mathrm{R}$ number); and it can be correlated with measures of social activity to model effectiveness of control measures such as comparing infection rates in schools, colleges and long term care facilities with the general population (Rhode Island department of health, USA). COVID-19 related data has been shared as open data all over the world and helped to build the FLOSS applications that use it. Open data and software supporting easy access to dashboards and visualizations are essential to counteract public uncertainty and distrust about infection control measures for this complex and confusing disease. However, ethics and data privacy should be considered for health data, such as contact tracing. Civic Technology movements are viewed as the collaboration of FLOSS use by citizens and open data by governments and offer a way to leverage the collective intelligence of human beings to overcome this pandemic.

Table 2 Association of COVID 19 dashboard deployment and open data Japanese prefectures.

\begin{tabular}{l|l|l|l|l|}
\hline & & \multicolumn{2}{|c|}{ COVID-19 Dashboard deployment } & \\
\hline & & Yes & No & Total \\
\hline & Open data & 20 & 2 & 22 \\
\hline $\begin{array}{l}\text { COVID-19 } \\
\text { related data }\end{array}$ & Not open data & 10 & 15 & 25 \\
\hline & Total & 10 & 17 & 47 \\
\hline
\end{tabular}

\section{References}

1. WHO Director-General's opening remarks at the media briefing on COVID-19 - 11 March 2020 [Internet]. [cited $2020 \mathrm{Jul}$ 9]. Available from: https://www.who.int/dg/speeches/detail/who-director-general-s-opening-remarks-at-the-mediabriefing-on-covid-19---11-march-2020

2. WHO Coronavirus Disease (COVID-19) Dashboard | WHO Coronavirus Disease (COVID-19) Dashboard [Internet]. [cited 2020 Oct 4]. Available from: https://covid19.who.int/

3. Risk communication - A moving target in the fight against infectious hazards and epidemics. Wkly Epidemiol Rec 2016 Feb 19;91(7):82-7.

4. Chang A, Schnall AH, Law R, Bronstein AC, Marraffa JM, Spiller HA, et al. Cleaning and Disinfectant Chemical Exposures and Temporal Associations with COVID-19 - National Poison Data System, United States, January 1, 2020-March 31, 2020. MMWR Morb Mortal Wkly Rep 2020 Apr 24;69(16):496-8

5. ITU: No scientific basis between $5 \mathrm{G}$ and COVID-19 [Internet]. [cited 2020 Jun 8]. Available from: https://www.itu.int/en/Pages/COVID-19/5gcovid-19-statement.aspx

6. Organization WH. Managing Epidemics (Key Facts About Major Deadly Diseases). $1^{\text {st }} \mathrm{ed}$. World Health Organization; 2018.

7. The Lancet Infectious Diseases. The COVID-19 infodemic. Lancet Infect Dis $2020 \mathrm{Jul}$ 17;20(8):875.

8. Pennycook G, McPhetres J, Zhang Y, Lu JG, Rand DG. Fighting COVID-19 Misinformation on Social Media: Experimental Evidence for a Scalable Accuracy-Nudge Intervention. Psychol Sci 2020 Jun 30;31(7):770-80.

9. Myth busters [Internet]. [cited 2020 Jun 10]. Available from: https://www.who.int/emergencies/ diseases/novel-coronavirus-2019/advice-for-public/myth-busters

10. Islam MS, Sarkar T, Khan SH, Mostofa Kamal A-H, Hasan SMM, Kabir A, et al. COVID-19-Related Infodemic and Its Impact on Public Health: A Global Social Media Analysis. Am J Trop Med Hyg 2020 Aug 10;

11. Reducing Stigma $\mid$ CDC [Internet]. [cited 2020 Jun 10]. Available from: https://www.cdc.gov/ coronavirus/2019-ncov/daily-life-coping/reduc- ing-stigma.html

12. Kobayashi S, Kane TB, Paton C. The privacy and security implications of open data in healthcare. Yearb Med Inform 2018 Apr 22;27(1):41-7.

13. Paton C, Kobayashi S. An open science approach to artificial intelligence in healthcare. Yearb Med Inform 2019 Aug;28(1):47-51.

14. Karopka T, Schmuhl H, Marcelo A, Dal Molin J, Wright G. Towards Open Collaborative Health Informatics - The Role of Free/Libre Open Source Principles. Yearb Med Inform 2011 Aug;20(01):63-72.

15. Paton C, Karopka T. The role of free/libre and open source software in learning health systems. Yearb Med Inform 2017 Aug;26(1):53-8.

16. Kobayashi S, Yahata K, Goudge M, Okada M, Nakahara T, Ishihara K. Open source software in medicine and its implementation in Japan. Journal on Information Technology in Healthcare 2009; 7(2):95-101.

17. GNU Health $\mid$ Freedom and Equity in Healthcare [Internet]. [cited 2021 Jan 21]. Available from: https://www.gnuhealth.org/about-us.html

18. Dehnavieh R, Haghdoost A, Khosravi A, Hoseinabadi F, Rahimi H, Poursheikhali A, et al. The District Health Information System (DHIS2): A literature review and meta-synthesis of its strengths and operational challenges based on the experiences of 11 countries. Health Inf Manag 2019 May;48(2):62-75.

19. OpenMRS Annual Report 2019 - Community OpenMRS Talk [Internet]. [cited 2020 Nov 2]. Available from: https://talk.openmrs.org/t/openmrs-annual-report-2019/27530

20. LIFE: Life-Saving Instruction for Emergencies Medical Simulation Training App - A simulation training app for emergency medicine and paediatrics [Internet]. [cited 2021 Jan 24]. Available from: https://oxlifeproject.org/

21. Noticia |Covid-19: [Follow-up of suspicious cases, through a computer module] seguimiento de casos sospechosos, a través de un módulo informático [Internet]. [cited 2020 Oct 12]. Available from: https://diamante.gob.ar/prensa/covid-19-seguimiento-de-casos-sospechosos-a-travs-de-un-mdulo-informtico-N5100

22. [Who are the 64 Argentine scientists chosen to finance their research on Covid-19] Quienes son los 64 científicos argentinos elegidos para financiar sus investigaciones sobre Covid-19 - Infobae 
[Internet]. [cited 2020 Oct 12]. Available from: https://www.infobae.com/coronavirus/2020/05/02/ quienes-son-los-64-cientificos-argentinos-elegidos-para-financiar-sus-investigaciones-sobre-covid-19/

23. Tackling the Beast: Using GNU Health to help the fight against the COVID-19 pandemic - GFOSS - Open Technologies Alliance [Internet]. [cited 2020 Aug 31]. Available from: https://gfoss.eu/ tackling-the-beast-using-gnu-health-to-help-thefight-against-the-covid-19-pandemic/

24. The European Commission's EUvsVirus Hackathon: The EUvsVirus Hackathon is a European Commission initiative that is open to EVERYONE AROUND THE WORLD on April 24-26. Devpost [Internet]. [cited 2020 Oct 12]. Available from: https://eunitedvsvirus.devpost.com/

25. Mamlin BW, Biondich PG, Wolfe BA, Fraser H, Jazayeri D, Allen C, et al. Cooking up an open source EMR for developing countries: OpenMRS - a recipe for successful collaboration. AMIA Annu Symp Proc 2006;529-33.

26. Oza S, Jazayeri D, Teich JM, Ball E, Nankubuge PA, Rwebembera J, et al. Development and Deployment of the OpenMRS-Ebola Electronic Health Record System for an Ebola Treatment Center in Sierra Leone. J Med Internet Res 2017 Aug 21;19(8):e294.

27. Kaphle S, Chaturvedi S, Chaudhuri I, Krishnan R, Lesh N. Adoption and Usage of mHealth Technology on Quality and Experience of Care Provided by Frontline Workers: Observations From Rural India. JMIR Mhealth Uhealth 2015 May 28;3(2):e61.

28. MedicMobile Community Health Toolkit [Internet]. [cited 2020 Nov 5]. Available from: http:// medicmobile.org/tools

29. Open Data Kit [Internet]. [cited 2020 Nov 2]. Available from: https://opendatakit.org/

30. Open Concept Lab' ' [Internet]. [cited 2020 Nov 3]. Available from: https://digitalhealthatlas.org/ en/-/projects/661/published

31. Baskaya M, Yuksel M, Erturkmen GBL, Cunningham M, Cunningham P. Health4Afrika - Implementing HL7 FHIR Based Interoperability. Stud Health Technol Inform 2019 Aug 21;264:20-4.

32. Updating NepalEHR in response to COVID19 [Internet]. [cited 2020 Nov 3]. Available from: https:// nepalehr.atlassian.net/wiki/spaces/NEPALEHR/ blog/2020/03/25/43090009/Updating+NepalEH$\mathrm{R}+$ in + response + to + COVID19

33. Muinga N, Paton C. Virtual reality for medical and nursing training in low- and middle-income countries. Pathways for Prosperity Commission Background Paper Series; 2019.
34. Tuti T, Winters N, Edgcombe H, Muinga N, Wanyama C, English M, et al. Evaluation of Adaptive Feedback in a Smartphone-Based Game on Health Care Providers' Learning Gain: Randomized Controlled Trial. J Med Internet Res $2020 \mathrm{Jul}$ 6;22(7):e17100.

35. Ferretti L, Wymant C, Kendall M, Zhao L, Nurtay A, Abeler-Dörner L, et al. Quantifying SARS$\mathrm{CoV}-2$ transmission suggests epidemic control with digital contact tracing. Science 2020 May $8 ; 368(6491)$

36. Show evidence that apps for COVID-19 contact-tracing are secure and effective. Nature 2020;580(7805):563.

37. Ethical considerations to guide the use of digital proximity tracking technologies for COVID-19 contact tracing [Internet]. [cited 2020 Jul 28]. Available from: https://www.who.int/publications/i/item/WHO-2019-nCoV-Ethics_Contact_ tracing apps-2020.1

38. Covid-19Radar/Covid19Radar: Open Source / Internationalization/iOS Android Cross Platform Contact Tracing App by exposure notification framework Xamarin App and Server Side Code [Internet]. [cited 2020 Aug 11]. Available from: https:/github.com/Covid-19Radar/Covid19Radar

39. Reintjes R. Lessons in contact tracing from Germany. BMJ 2020 Jun 25;369:m2522.

40. Li M, Leslie H, Qi B, Nan S, Feng H, Cai H, et al. Development of an openEHR Template for COVID-19 Based on Clinical Guidelines. J Med Internet Res 2020 Jun 10;22(6):e20239.

41. The Open Data Handbook [Internet]. [cited 2020 Oct 21]. Available from: https://opendatahandbook.org/guide/en/

42. Martin EG, Begany GM. Opening government health data to the public: benefits, challenges, and lessons learned from early innovators. J Am Med Inform Assoc 2017 Mar 1;24(2):345-51.

43. Huston P, Edge VL, Bernier E. Reaping the benefits of Open Data in public health. Can Commun Dis Rep 2019 Oct 3;45(11):252-6.

44. D'Agostino M, Samuel NO, Sarol MJ, de Cosio FG, Marti M, Luo T, et al. Open data and public health. Rev Panam Salud Publica 2018 Jun 5;42:e66.

45. Yozwiak NL, Schaffner SF, Sabeti PC. Data sharing: Make outbreak research open access. Nature 2015 Feb 26;518(7540):477-9.

46. Kieny M-P, Moorthy V, Bagozzi D. Public health: Use open data to curb Zika virus. Nature 2016 May 26;533(7604):469.

47. Zhao L, Feng D, Ye R-Z, Wang H-T, Zhou Y-H, Wei J-T, et al. Outbreak of COVID-19 and SARS in mainland China: a comparative study based on national surveillance data. BMJ Open 2020 Oct 15;10(10):e043411.

48. Severe acute respiratory syndrome coronavirus 2 isolate Wuhan-Hu-1, co - Nucleotide - NCBI [Internet]. [cited 2020 Jul 8]. Available from: https:// www.ncbi.nlm.nih.gov/nuccore/MN908947

49. Roser M, Ritchie H, Ortiz-Ospina E, Hasell J. Coronavirus Pandemic (COVID-19) - Statistics and Research - Our World in Data. Our World in Data 2020 Mar 4;

50. Open-Access Data and Computational Resources to Address COVID-19 | Data Science at NIH [Internet]. [cited 2021 Jan 24]. Available from: https://datascience.nih.gov/covid-19-open-accessresources

51. Dong E, Du H, Gardner L. An interactive webbased dashboard to track COVID-19 in real time. Lancet Infect Dis 2020 Feb 19;20(5):533-4.

52. Lin C, Braund WE, Auerbach J, Chou J-H, Teng $\mathrm{J}-\mathrm{H}, \mathrm{Tu}$ P, et al. Policy Decisions and Use of Information Technology to Fight COVID-19, Taiwan. Emerging Infect Dis $2020 \mathrm{Jul} ; 26(7): 1506-12$.

53. Taiwan's battle against coronavirus began in late 2019 - Nikkei Asian Review [Internet]. [cited 2020 Jul 13]. Available from: https://asia.nikkei.com/ Spotlight/Coronavirus/Taiwan-s-battle-againstcoronavirus-began-in-late-2019

54. Singapore's police now have access to contact tracing data $\mid$ MIT Technology Review [Internet]. [cited 2021 Jan 20]. Available from: https://www. technologyreview.com/2021/01/05/1015734/ singapore-contact-tracing-police-data-covid/

55. TraceTogether [Internet]. [cited 2021 Jan 21]. Available from: https://www.tracetogether.gov.sg/ common/privacystatement/

56. Reddick CG, editor. Comparative E-Government. vol. 25. New York, NY: Springer; 2010.

57. Yuan EJ, Hsu C-A, Lee W-C, Chen T-J, Chou L-F, Hwang S-J. Where to buy face masks? Survey of applications using Taiwan's open data in the time of coronavirus disease 2019. J Chin Med Assoc 2020;83(6):557-60.

58. WorldHealthOrganization/app: COVID-19 App [Internet]. [cited 2020 Nov 10]. Available from: https://github.com/WorldHealthOrganization/app

\section{Correspondence to:}

Shinii Kobayashi

2-3-6, Minami, Wako

Saitama, 351-0197

Japan

E-mail:skoba@moss.gr.jp 